\title{
Intestinal Pseudoobstruction Caused by Chronic Lyme Neuroborreliosis. A Case Report
}

\author{
David F Schefte* and Tyge Nordentoft \\ Department of Gastrointestinal Surgery, Hvidovre Hospital, University of Copenhagen, Hvidovre, Denmark
}

Chronic intestinal pseudoobstruction is often classified as idiopathic. The condition is associated with poor quality of life and high morbidity, and treatment options are often unsatisfactory. A case of chronic intestinal pseudoobstruction in a 66-year-old woman, presenting with back and abdominal pain, urinary retention and severe constipation is described. The patient lived in an area in which Lyme disease is endemic and had been bitten by ixodes ticks. Intrathecal synthesis of anti-borrelia IgM and IgG and lymphocytosis in the cerebrospinal fluid was found, consistent with chronic Lyme neuroborreliosis since symptoms had lasted for more than six months. The patient's gastrointestinal function recovered and the pain subsided significantly following treatment with antibiotics. Lyme neuroborreliosis (LNB) often results in palsy, but rarely affects the autonomic nervous system. Three patients have been described with intestinal pseudoobstruction due to acute LNB. However, this is the first described case of intestinal pseudoobstruction due to chronic Lyme neuroborreliosis. LNB must be suspected in patients with intestinal pseudoobstruction, in particular in patients who have been bitten by an ixodes tick and in patients living in an endemic area.

(J Neurogastroenterol Motil 2015;21:440-442)

Key Words

Constipation; Intestinal pseudoobstruction; Lyme neuroborreliosis

\section{Introduction}

Chronic constipation is a common disease in the Western world, especially among the elderly population. ${ }^{1}$ This condition can usually be treated conservatively with lifestyle changes and laxatives. ${ }^{2}$ Nonetheless, occasionally patients with no previous history of gastrointestinal disease develop severe chronic constipation, often refractory to conservative treatment. This condition is also called chronic intestinal pseudoobstruction (CIP) and is often classified as idiopathic. ${ }^{3}$ Since the pathogenesis in some of these cases is due to a neuropathy involving the enteric nervous system, diseases that involve autonomic neuropathy, eg, diabetes mellitus, alcohol abuse, and paraneoplastic syndrome, are known causes of $\mathrm{CIP}^{3}$

In this case report we describe a case of sudden onset CIP due to chronic Lyme neuroborreliosis (LNB), which is known to cause neurological disorders, but has never before been described as the cause of CIP.

Received: October 9, 2014 Revised: January 9, 2015 Accepted: January 12, 2015

(c) This is an Open Access article distributed under the terms of the Creative Commons Attribution Non-Commercial License (http://creativecommons. org/licenses/by-nc/4.0) which permits unrestricted non-commercial use, distribution, and reproduction in any medium, provided the original work is properly cited.

${ }^{*}$ Correspondence: David F Schefte, MD

Department of Gastrointestinal Surgery, Hvidovre Hospital, University of Copenhagen, Kettegaard alle 30, 2650 Hvidovre, Denmark Tel: +45-21679544, E-mail: davidschefte@hotmail.com

Financial support: None.

Conflicts of interest: None.

Author contributions: Tyge Nordentoft has contributed to all elements in the making of the manuscript.

ORCID: David F Schefte, http://orcid.org/0000-0002-6532-9259. 


\section{Case Report}

A 66-year-old woman, with no major medical history was hospitalized due to urinary retention and lower back-pain radiating to the right lower extremity and lower abdomen. The patient lived in an area where Lyme disease is endemic and had been bitten by ixodes ticks previously. Physical examination on admission, including clinical neurological examination, was normal. Blood tests, including white blood cell count and C-reactive protein, were normal.

Spinal MRI revealed a suspected right-sided paramedian disc prolapse at L4/L5 with impingement of the L5 nerve root and a small disc prolapse at L5/S1. This was treated conservatively with physiotherapy and analgesia, including opioids. While the urinary retention resolved spontaneously, the pain intensified and the patient developed severe constipation, unresponsive to laxatives. Abdominal X-ray and CT scan suspected colonic ileus with some dilation of the colon (Figure). Absence of intestinal obstruction was confirmed by barium X-ray and the condition was interpreted as intestinal pseudoobstruction resulting from opioid therapy. Treatment with an acetylcholinesterase inhibitor resulted in defecation and flatulence, but the effects were only temporary. Colonoscopy revealed a flaccid, poorly-prepared bowel, but no other pathology was found. Colonic transit time investigation

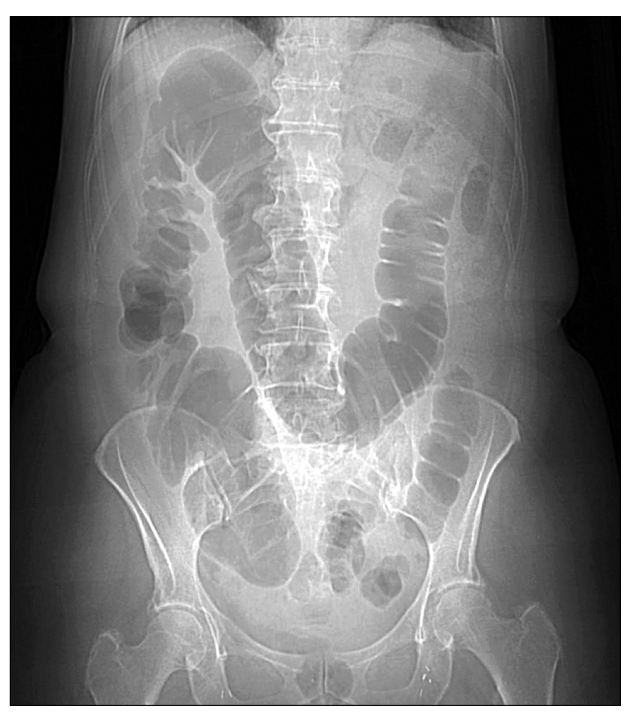

Figure. Abdominal X-ray of the patient with chronic intestinal pseudoobstruction due to chronic Lyme neuroborreliosis showed colonic ileus with dilation of the colon. Subsequent barium X-ray excluded intestinal obstruction and the condition was interpreted as intestinal pseudoobstruction. showed significantly delayed colonic emptying. Treatment with laxatives was intensified and transanal irrigation initiated.

More than 6 months after onset of symptoms the patient only defecated sporadically, even with twice daily transanal irrigation, supplemented with high dose osmotic and peristaltic stimulation. By this time she had lost $15 \mathrm{~kg}$.

As a result of persistent back pain a repeat clinical neurological assessment was performed, which remained normal. Lumbar puncture was performed and investigation of the cerebrospinal fluid (CSF) showed CSF lymphocytosis with spinal leucocyte count on $103 \times 10^{6} / \mathrm{L}, 100 \%$ mononuclear, elevated IgG-index, elevated total spinal protein content, positive oligoclonal bands and intrathecal borrelia antibody synthesis, IgG as well as IgM. Since the symptoms had lasted for more than 6 months this was consistent with chronic LNB. ${ }^{4}$ No other bacteria or malignant cells were found in the CSF. Anti-Bb IgM and IgG was found in the serum.

The patient received a course of intravenous ceftriaxone $2 \mathrm{~g}$ administered once daily for 3 weeks. The frequency of transanal irrigation was reduced 4 days after onset of antibiotic therapy and 2 weeks later she had daily defecation without transanal irrigation, with decreased use of laxatives. Following improvement in gastrointestinal function the patient experienced a decrease in pain. After 3 weeks of antibiotic therapy, intensity of the pain slowly subsided with subsequently reduced need for analgesia. Three months after antibiotic treatment the patient was having a daily bowel movement with no need for laxatives. The pain was now of low intensity (visual analog scale 1-2) when treated with secondary analgesics. In a repeated lumbar puncture, the spinal leucocyte count had decreased to $9 \times 10^{6} / \mathrm{L}$, spinal protein content had decreased and there was a dramatic decrease in intrathecal synthesis of anti-Bb IgM and IgG.

Subsequent nerve conduction studies showed no certain signs of myelopathy. The sensory evoked potentials showed bilateral peripheral affection of the upper extremities and the motor evoked potentials showed bilateral peripheral affection to musculus abductor pollicis brevis.

\section{Discussion}

This case report describes a case of CIP due to chronic LNB. LNB is a nervous system disorder caused by Borrelia burgdorferi (Bb), a tick-borne spirochete. Borreliosis is highly endemic in southern Scandinavia and central Europe. In North America, borreliosis occurs mainly in the North Eastern states but chronic 
LNB is almost solely reported in Europe. ${ }^{4}$

LNB is divided into early and chronic disease. Chronic LNB is defined as disease duration of more than six months, CSF inflammation and $\mathrm{Bb}$ specific intrathecal IgG synthesis. Less than $1-2 \%$ of patients with LNB develop chronic LNB. ${ }^{4}$

Borreliosis often presents as erythema migrans and if untreated approximately $5 \%$ will develop LNB in 2-6 weeks. The most common manifestation of early LNB is a subacute painful meningoradiculitis called Bannwarths syndrome and consists of CSF inflammation, painful radiculitis and palsies. Bannwarths syndrome comprises more than $80 \%$ of LNB cases but approximately $30 \%$ of these patients present without motor signs as seen in the present case. Characteristically, the pain is migrating, burning, often located in the back and radiating to the upper and lower extremities, abdomen and/or chest. Approximately 50\% have cranial nerve involvement, predominantly a peripheral facial nerve palsy.

In the chronic stage, patients typically present with headache, malaise, significant weight loss, sensorineural hearing loss or a progressive spastic-ataxic gait disturbance. ${ }^{4}$

Autonomic dysfunction is rarely seen in $\mathrm{LNB}^{4}$ If $\mathrm{LNB}$ is suspected, investigation of anti- $\mathrm{Bb}$ in the serum must be made. In the first stage of Lyme borreliosis, $20-50 \%$ of the patients will be anti-Bb IgM positive. Twelve weeks after onset of neurological symptoms, all patients will have detectable anti-Bb IgG in the serum. ${ }^{4}$ If the blood sample is positive for anti-Bb the CSF must be examined. Specific CSF antibodies appear during the second week after onset of neurological symptoms and are detectable in $100 \%$ of patients 8 weeks after onset. All patients have pronounced CSF inflammation with lymphocytosis and high protein content. Disease duration for more than 6 months, CSF inflammation and $\mathrm{Bb}$ specific intrathecal IgG synthesis is diagnostic of chronic LNB. ${ }^{4}$

Chronic LNB seems not to be self-limiting, but antibiotic therapy arrests disease progression and patients recover significantly. However, sequelae may occur. There is no evidence of improved outcome when treating LNB patients with antibiotics for more than 2 weeks. ${ }^{4}$

The patient in the present case, initially experienced radicular pain, but she also developed urinary retention and severe intestinal pseudoobstruction as manifestations of autonomic dysfunction.

The results of the nerve conduction studies were regarded as insignificant since the patient had no neurological symptoms of the upper extremities during the course of disease.

Autonomic dysfunction in LNB has been reported before.
Urinary retention in LNB is described previously. ${ }^{5-7}$ Intestinal pseudoobstruction in patients with acute LNB has been described in three patients, two of whom had symptomatology very similar to the patient in the present case, with urinary retention in addition to the intestinal pseudoobstruction. ${ }^{5,8}$

To our knowledge, the present case report is the first reported case of CIP due to chronic LNB.

CIP is associated with poor quality of life and high morbidity. ${ }^{3}$ Pathophysiological aspects of the condition remain obscure and treatment options are limited and often unsatisfactory. Consequently, it is crucial that all physicians and gastroenterologists in particular, are aware of the non-idiopathic causes, and especially those that can be treated successfully. Infection with $\mathrm{Bb}$ can manifest as CIP and all of the 3 patients described, with acute LNB and intestinal pseudoobstruction, experienced complete remission after 2-3 weeks of antibiotic therapy. In the present case, 3 weeks of antibiotic treatment was effective even though the patient had developed chronic LNB.

In conclusion, $\mathrm{Bb}$ infection must be suspected in patients with sudden onset, severe constipation or intestinal pseudoobstruction, in particular in patients with simultaneous emergence of symptoms such as palsies, radicular pain, arthritis, and myocarditis, and in patients living in an endemic area.

\section{References}

1. Higgins PD, Johanson JF. Epidemiology of constipation in North America: a systematic review. Am J Gastroenterol 2004;99:750-759.

2. Eswaran S, Guentner A, Chey WD. Emerging pharmacologic therapies for constipation-predominant irritable bowel syndrome and chronic constipation. J Neurogastroenterol Motil 2014;20:141-151.

3. Antonucci A, Fronzoni L, Cogliandro L, et al. Chronic intestinal pseudo-obstruction. World J Gastroenterol 2008;14:2953-2961.

4. Hansen K, Crone C, Kristoferitsch W. Lyme neuroborreliosis. Handb Clin Neurol 2013;115:559-575.

5. Shamim EA, Shamim SA, Liss G, Nylen E, Pincus JH, Yepes M. Constipation heralding neuroborreliosis: an atypical tale of 2 patients. Arch Neurol 2005;62:671-673.

6. Olivares JP, Pallas F, Ceccaldi M, et al. Lyme disease presenting as isolated acute urinary retention caused by transverse myelitis: an electrophysiological and urodynamical study. Arch Phys Med Rehabil 1995;76:1171-1172.

7. Puri BK, Shah M, Julu PO, Kingston MC, Monro JA. Urinary bladder detrusor dysfunction symptoms in lyme disease. Int Neurourol J 2013;17:127-129.

8. Chatila R, Kapadia CR. Intestinal pseudoobstruction in acute Lyme disease: a case report. Am J Gastroenterol 1998;93:1179-1180.

9. Di NG, Blandizzi C, Volta U, et al. Review article: molecular, pathological and therapeutic features of human enteric neuropathies. Aliment Pharmacol Ther 2008;28:25-42. 